\title{
A new paradigm in viral resistance
}

\author{
Autumn Ruiz ${ }^{1}$, Stephen J Russell ${ }^{1}$ \\ 'Department of Molecular Medicine, Mayo Clinic, Rochester, Minnesota 55905, USA \\ Cell Research (2012) 22:1515-1517. doi:10.1038/cr.2012.139; published online 2 October 2012
}

The rapid mutation of RNA viruses allows for the acquisition of resistance to drugs directly targeting viral proteins. Therefore, a novel approach to the development of antivirals centers on targeting host factors critical to viral replication. A recent report has brought to light the potential for RNA viruses to also develop resistance against compounds targeting crucial host factors, suggesting that a combination of drugs with various targets may be necessary for preventing resistance.

Enteroviruses are members of the Picornaviridae family, a diverse group of viruses composed of several wellknown human pathogens, including human rhinovirus (HRV), hepatovirus (hepatitis A virus), and enteroviruses (poliovirus [PV], coxsackievirus [CV], and echovirus). These viruses are small, non-enveloped, single-stranded, positive-sense RNA ((+)-RNA) viruses. Enterovirus infections can cause a variety of diseases ranging from mild or asymptomatic infections to more severe conditions including aggravation of respiratory tract diseases, meningitis, encephalitis, myocarditis, acute paralysis, neonatal sepsis, and pancreatitis [1-3]. Currently, there are very few treatments for the majority of these viruses, emphasizing the importance of developing potent, nontoxic

Correspondence: Stephen J Russell

E-mail: sjr@mayo.edu therapeutics. The pursuit for potential treatments has been facilitated by studies focused on understanding key components within the picornaviral life cycle (viral and nonviral) as well as high-throughput screening of novel inhibitors. These studies have led to the development of several broad-spectrum enterovirus inhibitors targeting various viral components, some of which are currently being investigated in clinical trials [3].

Unfortunately, genetic variability and the evolution of drug resistance represent significant barriers to the prevention, treatment and eradication of viral infections, especially for RNA viruses, which exhibit an extremely high mutation rate. The rise in incidence of drug resistance has prompted a shift in strategy from developing effectors against rapidly mutating viruses to targeting the more static cellular components critical for viral replication. Targeting host factors as a means of antiviral therapy is an attractive prospect as it could affect multiple genotypes while minimizing the potential for viral resistance. Using this approach, the identification of possible targets for an array of viruses is under pursuit and has led to the development of several promising compounds for the treatment of HIV and hepatitis $\mathrm{C}$ virus [HCV] [4-5]. In light of these results, the development of an antiviral arsenal that includes inhibitors of both viral and cellular factors for combination-based therapies may prove to be the most beneficial way of combating viral infections. Thus, understanding the mechanisms of viral replication is crucial for the discovery and development of novel therapeutics against viral and nonviral components.

Following entry of $(+)$-RNA viruses, the genome is released and the viral proteins are subsequently translated and processed into mature proteins. These peptides then go on to assemble progeny virions as well as synthesize and recruit viral (+)-RNA genomes for packaging. (+)-RNA viruses accomplish this by assembling replication complexes on intracellular membrane surfaces. The complexes are composed of various non-structural viral proteins; viral RNA, the viral-RNA-dependent RNA polymerase, and several host cell factors. These membrane rearrangements appear to provide optimal lipid and protein conditions for recruiting and sustaining the necessary machinery for viral replication [6]. While some commonalities have been observed between viruses in the specific cellular factors recruited to the sites of replication, the identification of all host proteins involved and their specific mechanism of function are still under investigation. Specific cellular factors used by several picornaviruses (such as $\mathrm{PV}$ and CVB3) and flaviviruses (such as HCV) have been identified and their mechanistic roles and potential as therapeutic targets are currently being evaluated. Of note is that a microenvironment rich in phosphatidylinositol-4-phosphate (PI4P), established through the recruitment of 
the phosphatidylinositol-4-kinase III $\alpha$ (PI4KIII $\alpha$ ) and/or III $\beta$ (PI4KIII $\beta$ ), is crucial for replication of several of these viruses [7-13]. Therefore, inhibitors targeting these components would offer broad-acting therapeutic potential.

In a paper recently published in Cell Research, van der Schaar et al. [14] provide a comprehensive analysis of CVB3 escape mutants that replicate efficiently in the presence of several potent antiviral drugs known to inhibit the kinase PI4KIII $\beta$, a key cellular cofactor for CVB3 replication [11, 13]. PI4P, found in Golgi-associated viral replication complexes, is the major product of the PI4KIII $\beta$ kinase, but the mechanism by which it enhances CVB3 replication has not yet been elucidated. Van der Shaar et al. [14] studied three drugs capable of inhibiting CVB3 replication (enviroxime, GW5074, and PIK93) and show convincingly that (i) all three drugs inhibit PI4KIII $\beta$; (ii) CVB3 replication is not inhibited by two of these drugs in cells expressing a drug-resistant PI4KIII $\beta$; (iii) point mutations in the viral $3 \mathrm{~A}$ protein confer drug resistance and (iv) in contrast to wild-type CVB3, the drug-resistant escape mutants of CVB3 are able to replicate in cells with low PI4KIII $\beta$, and hence low PI4P.

Based on their findings the authors propose that CVB3 mutants escape the antiviral drug effect by somehow circumventing their need for PI4P. If this interpretation is correct, then to our knowledge, this is the first documented example of a picornavirus mutating to bypass its dependency on a cellular protein that is targeted by an antiviral agent. Thus, van der Schaar et al. may have established an important new paradigm in viral drug resistance. However, there still exists the formal possibility that the mutations in the $3 \mathrm{~A}$ protein conferring resistance to these antiviral drugs are simply increasing its affinity for its natural substrate, thereby allowing the virus to propagate despite a reduction in the concentration of the cellular factor on which the $3 \mathrm{~A}$ protein depends. Additional work will therefore be needed to determine whether the drug-resistant viruses examined in this paper are truly able to replicate in the complete absence of PI4KIII $\beta$. Whatever the answer to that question, the sobering message of this very interesting study is that viruses can acquire resistance to antiviral drugs independently of whether the drug targets a viral enzyme or a host factor critical for viral replication.

If in fact these resistance mutations have resulted in a virus with a complete alteration in the cellular factors required for replication rather than an increase in binding affinity for a substrate, then the risk associated with using such antivirals may need to be reassessed. However, regardless of the specific mechanism of resistance, van der Schaar et al. have emphasized the potential need for combinatorial therapies when targeting host factors. Therefore, dissecting the pathways by which different viruses manipulate host factors in order to establish and complete replication is critical. The authors have demonstrated the advantages of identifying resistance mutants for studying the role of viral and cellular proteins in virus replication and deciphering the mechanisms of action for new antivirals. Previously, an $\mathrm{HCV}$ mutant resistant to Alisporivir and cyclosporine $\mathrm{A}$ (two antivirals targeting host factors critical to HCV replication) was identified [15]. The discovery of yet another mutant virus with the ability to replicate in the absence of high levels of a crucial host factor by van der Schaar et al. lends additional evidence for the validity of this phenomenon. Taken together, these studies emphasize the possibility that targeting host factors will inevitably lead to a similar clinical outcome as directly targeting viral proteins. The rate of resistance development may still be highly reduced by host-specific inhibitors; however, the identification and development of combinatorial therapies (potentially targeting crucial host and viral compo- nents) prior to clinical translation may prove to be more efficient. In any case, the importance of fully understanding the mechanisms by which individual viruses and compound-resistant viruses manipulate host cellular proteins with regard to treatment has become increasingly apparent.

\section{References}

1 Gern JE. The ABCs of rhinoviruses, wheezing, and asthma. J Virol 2010; 84:7418-7426.

2 Tebruegge M, Curtis N. Enterovirus infections in neonates. Semin Fetal Neonatal Med 2009; 14: 222-227.

3 Thibaut HJ, De Palma AM, Neyts J. Combating enterovirus replication: state-of-the-art on antiviral research. Biochem Pharmacol 2012; 83:185192.

4 Gilliam BL, Riedel DJ, Redfield RR. Clinical use of CCR5 inhibitors in HIV and beyond. J Transl Med 2011; 9 Suppl 1:S9.

5 von Hahn T, Ciesek S, Manns MP. Arrest all accessories-inhibition of hepatitis $\mathrm{C}$ virus by compounds that target host factors. Discov Med 2011; 12:237244.

6 Miller S, Krijnse-Locker J. Modification of intracellular membrane structures for virus replication. Nat Rev Microbiol 2008; 6:363-374.

7 Tai AW, Benita Y, Peng LF, et al. A functional genomic screen identifies cellular cofactors of hepatitis $\mathrm{C}$ virus replication. Cell Host Microbe 2009; 5:298-307.

8 Vaillancourt FH, Pilote L, Cartier M, et al. Identification of a lipid kinase as a host factor involved in hepatitis $\mathrm{C}$ virus RNA replication. Virology 2009; 387:510.

9 Berger KL, Cooper JD, Heaton NS, et al. Roles for endocytic trafficking and phosphatidylinositol 4-kinase III alpha in hepatitis C virus replication. Proc Natl Acad Sci USA 2009; 106:75777582.

10 Borawski J, Troke P, Puyang X, et al. Class III phosphatidylinositol 4-kinase alpha and beta are novel host factor regulators of hepatitis $\mathrm{C}$ virus replication. J Virol 2009; 83:10058-10074.

11 Hsu NY, Ilnytska O, Belov G, et al. Vi- 
ral reorganization of the secretory pathway generates distinct organelles for RNA replication. Cell 2010; 141:799811.

12 Reiss S, Rebhan I, Backes P, et al. Recruitment and activation of a lipid kinase by hepatitis C virus NS5A is essential for integrity of the membranous replication compartment. Cell Host Microbe 2011; 9:32-45.
13 Arita M, Kojima H, Nagano T, Okabe T, Wakita T, Shimizu H. Phosphatidylinositol 4-kinase III beta is a target of enviroxime-like compounds for antipoliovirus activity. J Virol 2011; 85:2364-2372.

14 van der Schaar HM, van der Linden L, Lanke KHW, et al. Coxsackievirus mutants that can bypass host factor PI4KIII $\beta$ and the need for high levels of PI4P lipids for replication. Cell Res 2012; 22:1576-1592.

15 Coelmont L, Hanoulle X, Chatterji U, et al. DEB025 (Alisporivir) inhibits hepatitis $\mathrm{C}$ virus replication by preventing a cyclophilin A induced cis-trans isomerisation in domain II of NS5A. PLoS One 2010; 5:e13687. 